\title{
A diagnostic interface for the ICOsahedral Non-hydrostatic (ICON) modelling framework based on the Modular Earth Submodel System (MESSy v2.50)
}

\author{
Bastian Kern and Patrick Jöckel \\ Deutsches Zentrum für Luft- und Raumfahrt e.V. (DLR), Institut für Physik der Atmosphäre, Oberpfaffenhofen, Germany \\ Correspondence to: Bastian Kern (bastian.kern@dlr.de)
}

Received: 18 May 2016 - Published in Geosci. Model Dev. Discuss.: 20 June 2016

Revised: 2 September 2016 - Accepted: 21 September 2016 - Published: 13 October 2016

\begin{abstract}
Numerical climate and weather models have advanced to finer scales, accompanied by large amounts of output data. The model systems hit the input and output (I/O) bottleneck of modern high-performance computing (HPC) systems. We aim to apply diagnostic methods online during the model simulation instead of applying them as a postprocessing step to written output data, to reduce the amount of I/O. To include diagnostic tools into the model system, we implemented a standardised, easy-to-use interface based on the Modular Earth Submodel System (MESSy) into the ICOsahedral Non-hydrostatic (ICON) modelling framework. The integration of the diagnostic interface into the model system is briefly described.

Furthermore, we present a prototype implementation of an advanced online diagnostic tool for the aggregation of model data onto a user-defined regular coarse grid. This diagnostic tool will be used to reduce the amount of model output in future simulations.

Performance tests of the interface and of two different diagnostic tools show, that the interface itself introduces no overhead in form of additional runtime to the model system. The diagnostic tools, however, have significant impact on the model system's runtime. This overhead strongly depends on the characteristics and implementation of the diagnostic tool. A diagnostic tool with high inter-process communication introduces large overhead, whereas the additional runtime of a diagnostic tool without inter-process communication is low. We briefly describe our efforts to reduce the additional runtime from the diagnostic tools, and present a brief analysis of memory consumption. Future work will focus on optimisation of the memory footprint and the I/O operations of the diagnostic interface.
\end{abstract}

\section{Introduction}

The resolution of numerical climate and weather simulations has advanced to finer scales, driven by increasingly potent high-performance computing (HPC) systems. Throughput of high-resolution simulations on modern multi-core systems increases, accompanied by large amounts of output data. These huge datasets pose challenges to the developer and the user, e.g. on how to cope with limited input and output (I/O) resources during data production, handling, processing, and archiving. Thus, the "workflow", i.e. how data produced by the model is handled, stored, and processed in subsequent steps, becomes more critical.

The performance of present-day HPC systems reaches the Petascale, i.e. providing peak performances of more than $10^{15}$ operations per second (see http://top500.org/). While the increase in computational power still follows Moore's law, this does not hold for the performance of the $\mathrm{I} / \mathrm{O}$ (sub-)system. I/O operations are limited by the bandwidth the I/O system offers to the compute system. With increasing computational power, the ratio between $\mathrm{I} / \mathrm{O}$ bandwidth and floating point operations per second decreases. Thus, for many applications the main bottleneck is $\mathrm{I} / \mathrm{O}$, potentially impacting the application performance on the next generation of supercomputers (Ali et al., 2009). Furthermore, storage capacity grows at a smaller rate as the computational speed (Kunkel et al., 2014; Kuhn et al., 2016). These problems especially persist for data intensive applications like numerical climate models, relying on fast data throughput and large storage capacity.

An obvious solution addressing the problems of data handling, the I/O performance gap, and the limited storage ca- 
pacity is to reduce the amount of output data. But a simple spatial and temporal reduction (coarse graining) may prove counterproductive. When we apply post-processing tools to the coarsened datasets, it deteriorates advantages gained through finer-resolved numerical simulations.

Recent studies on volume reduction of climate data focus on lossless and lossy compression of data online or as post-processing (Kuhn et al., 2016; Baker et al., 2016). We propose an approach of using diagnostic analyses, which are usually applied on the data after they are stored on disk, online during the model simulation. Depending on the analyses needed and selected, with the online diagnostic tools we might be able to drop parts of the output of variables on the full model resolution. This reduces data transfer over the computer network and disk storage occupation. The application of online diagnostic tools enables us to use the fully resolved data, to perform data reduction, and even to apply advanced diagnostics, which are not applicable offline. Online diagnostic tools can also be combined with any kind of data compression.

Such online diagnostics can be very simple and the implementation into the model system code can be straightforward. In most cases, however, the direct implementation of diagnostic tools in the model introduces dependencies throughout the code, which will be problematic with respect to maintenance of both the model system and the diagnostic tool itself. This problem grows larger with the number of model variables accessed by the advanced diagnostic tool and the complexity of its code structure.

A direct implementation of diagnostics may also be difficult for users, who apply the model system but have low experience with the model code itself. We know from experience that, e.g. adding new variables to the model system or finding the right place to plug-in your diagnostic tool sometimes turns out to be cumbersome. Furthermore, unexperienced developers may not be aware of possible side effects they introduce to the model system code and their impact on the model performance. A standardised, well-documented, and easy-to-use interface supports users during the implementation of their diagnostic tools and helps to disentangle developments of the tools and the model system itself.

In this study, we present a first application of an interface for online diagnostic tools based on the Modular Earth Submodel System (MESSy v2.50; Jöckel et al., 2010) in the ICOsahedral Non-hydrostatic model framework (ICON; Zängl et al., 2015). In Sect. 2 we briefly describe the model system, the structure of the interface, and the implementation of the diagnostic interface into the model system. Then we present a prototype implementation of an advanced diagnostic tool for data aggregation on a user-defined regular coarse grid (Sect. 3). The performance of the diagnostic interface and two different online diagnostic tools is analysed in Sect. 4. In the end we present ongoing developments and future plans for applications (Sect. 5) and close with our conclusions (Sect. 6).

\section{Model system}

The work described here is part of the German-wide $\mathrm{HD}(\mathrm{CP})^{2}$ (High Definition Clouds and Precipitation for advancing Climate Prediction; http://hdcp2.eu/) research initiative. $\mathrm{HD}(\mathrm{CP})^{2}$ is funded by the German Federal Ministry of Education and Research (BMBF) and aims to improve our understanding of clouds and precipitation and their implication for climate prediction. The research attempt follows an integrated "three-pillar" approach combining model development and observations via a synthesis group.

$\mathrm{HD}(\mathrm{CP})^{2}$ focuses on the development of a high-resolved model system and its application for simulations with a horizontal grid-spacing of about $100 \mathrm{~m}$ (Dipankar et al., 2015). To evaluate this model system on fine-resolved scales, highresolution observational datasets were obtained during the $\mathrm{HD}(\mathrm{CP})^{2}$ Observational Prototype Experiment (HOPE) between April and May 2013 (see Buehler and Russchenberg, 2014). In the synthesis project, model and observational data are analysed and combined to evaluate and improve parameterisations in climate models.

One goal of the synthesis project is to develop advanced diagnostics and forward operators applicable to both, observations and model data. This approach of consistently processing data from observations and from model simulations enhances comparability between both. For this, we implemented a dedicated interface for the integration of enhanced diagnostic operators into the model system. We aim to provide a generalised and easy-to-use interface to plug-in existing and novel diagnostic tools for application within ICON. The use of a dedicated diagnostic interface in ICON, furthermore, supports concurrent developments of the diagnostic tools and of the model system, which is still further enhanced during the project. A generalised interface also enhances the possibility of using the same diagnostic tools with other numerical models. Next, we will give a short overview of the involved components, before we present the implementation details of the diagnostic interface in the model system. Although, this study focuses on the implementation in ICON, the implementation in other numerical models would be similar.

\subsection{ICON}

The ICON (Zängl et al., 2015) is actively developed at the German Weather Service (DWD, Deutscher Wetterdienst) and the Max Planck Institute for Meteorology (MPI-M). The joint project targets a unified modelling system for application in global numerical weather prediction and climate modelling. Three main goals were defined during the initial phase, driving the implementation of the non-hydrostatic core of the model system (Zängl et al., 2015). These goals are

- the achievement of better conservation properties compared to the existing global model systems at the insti- 
tutions, i.e. local mass conservation and mass-consistent transport, and the wish for energy conservation;

- the scalability of the model system on future parallel HPC platforms;

- the availability of static mesh refinement, evolved during the model development into the capability of multiple one-way and two-way nested grids within one model application and an option for vertical nesting.

The model system is discretised on an unstructured triangular C-grid, derived from a spherical icosahedron using iterative refinement. Discretisation on this grid does not support exact global energy conservation, but the triangular grid was chosen because of the more convenient implementation of nesting using an hierarchical mesh refinement. Details on the discretisation of the equations of motion on the triangular $\mathrm{C}$-grid and the numerical implementation of the non-hydrostatic dynamical core are described by Zängl et al. (2015).

\subsection{MESSy}

The MESSy (http://www.messy-interface.org/; Jöckel et al., 2005,2010 ) is a framework for the implementation of (parts of) Earth system models (ESMs). It provides a standardised and generalised interface for the implementation and coupling of multiple ESM components (e.g. dynamical cores, physical parameterisations, chemistry packages, diagnostic tools) called "submodels". Currently, there are more than 60 submodels available, covering general infrastructure, diagnostics, atmospheric chemistry, and model physics. The MESSy interface was integrated into various numerical models (ECHAM5, Jöckel et al., 2005, 2010; COSMO, Kerkweg and Jöckel, 2012a; CLaMS, Hoppe et al., 2014; CESM, Baumgaertner et al., 2016; see glossary for acronyms).

In geoscientific modelling, coupling of multi-institutional codes with generally different domain decompositions is a widely used approach for building model systems. In general, either external couplers or frameworks for internal coupling are applied. An extended classification of coupling methods can be found in Appendix A of Kerkweg and Jöckel (2012b) and in Jöckel (2012). An overview of different coupling techniques in Earth system modelling is presented by Valcke et al. (2012). Common external couplers in the Earth system model community are, e.g. OASIS3 (Valcke et al., 2006; Valcke, 2013), OASIS4 (Redler et al., 2010), and CPL6 (Craig et al., 2005), as used in the Community Climate System Model version 3 (CCSM3; Collins et al., 2006). Widely used examples for internal coupling are the Earth System Modeling Framework (ESMF; Collins et al., 2005) and the Community Climate System Model version 4 (CCSM4; Gent et al., 2011). This approach is also used in space weather modelling with the Space Weather Modeling Framework (SWMF; Tóth et al., 2005). Recently, Hanke et al. (2016) developed the Clibrary YAC (Yet Another Coupler), which provides paral- lelised and efficient algorithms for grid transformation, interpolation, and data exchange.

In contrast to the coupling of "domains", MESSy was originally developed to work on the same spatial domain and parallel domain decomposition as the base model, applying a formalised process-based operator splitting (Jöckel et al., 2005). The original implementation was intended to equip the atmospheric general circulation model ECHAM5 (Roeckner et al., 2006) with additional processes for atmospheric chemistry (EMAC, ECHAM5/MESSy Atmospheric Chemistry model; Jöckel et al., 2006, 2010). Operator splitting as an internal coupling method is implemented (implicitly and less formalised) in the numerical model codes anyway, to integrate the different processes. However, the operator splitting approach of MESSy proves more powerful, also allowing for coupling of different domains, e.g. demonstrated by the integration of an ocean subsystem (Pozzer et al., 2011). An extension by Kerkweg and Jöckel (2012b) allowed for one-way coupling of different spatially nested domains using a server-client approach with point-to-point communication. Extension into a two-way nested atmospheric chemistry model system is currently under development.

The concept of the MESSy interface consists of a four layer structure for the integration of submodels. This ensures the disentanglement between infrastructure and submodels. The "base model layer" (BML) consists of a central clock and runtime control. In current implementations, the part of the base model is provided by a general circulation model, a numerical weather model, or a box model, extended by standardised calls to MESSy-specific main entry routines. The "base model interface layer" (BMIL) provides the standardised entry points to plug-in submodels. This part is base model specific, as it provides the additional infrastructure of MESSy. The "submodel interface layer" (SMIL) connects the specific implementation of the process to the infrastructure in the BMIL. The specific implementation of the process resides in the "submodel core layer" (SMCL). In this layout, the BMIL and SMIL could be seen as layers, translating between the base model and the specific implementation of the submodel.

Standardisation of the connection between BMIL and SMIL ensures separation of developments in the base model and in submodels. Using MESSy in a new base model requires a one-time implementation of a base model-specific BMIL. Furthermore, for each new submodel a SMIL module has to be implemented. From the SMIL any legacy routines in the submodel core can be called, which opens the ability to reuse existing code. Porting submodels to different base models thus becomes straightforward. Only sometimes, this requires minimal generalisation in the SMIL, when the submodel was initially designed for a specific base model.

The user interface of the MESSy framework is based on Fortran95 namelists, offering control over infrastructure and submodels, including the possibility to (de)activate submod- 
els and to change submodel-specific parameters. This minimises the need for re-compilation of the model system when changing the combination of submodels and/or parameter values between different simulations.

\subsection{Integration of MESSy into ICON}

The implementation of the MESSy interface in ICON follows the MESSy standard. Calls in the ICON code to subroutines in the MESSy BMIL are enclosed in preprocessor directives (\#ifdef MESSY ... \# \#ndif). This allows a strict separation of ICON and MESSy code, and guarantees the compilation of the ICON model without any missing dependencies, if the MESSy code is not included. Base model-specific code is avoided in the MESSy interface, but when necessary, it is also enclosed in preprocessor directives (\#ifdef ICON ... \# \#ndif). To use the MESSy interface, the user just has to invoke the standard ICON configuration script with an optional parameter string (./configure --with-messy) and has to make sure, that the MESSy code is available in the model distribution under externals/messy.

We are still actively developing and extending the code, regularly merging developments from the main development branch of ICON and development branches of the $\operatorname{HD}(C P)^{2}$ project. Due to the four layer structure, only sometimes minimal changes in the MESSy BMIL code are necessary to ensure a working model system including the diagnostic interface.

The output infrastructure of the diagnostic interface was extended to use the CDI (Climate Data Interface; https: //code.zmaw.de/projects/cdi) library to provide data output consistent with the ICON base model. However, the interface does not support asynchronous data output. Currently, the diagnostic interface provides only strict serial output to the diagnostic submodels. This is a very strong limitation, which will be removed soon, as a parallel version of CDI is being developed at Deutsches Klimarechenzentrum (DKRZ; German Climate Computing Centre). As we will see in the performance tests, the limited output capabilities of the diagnostic interface have quite an impact on runtime. However, this impact is limited and we provide comparisons without any data output, to estimate the impact of the diagnostic calculations on the model system runtime.

The implementation as diagnostic interface in ICON is based on MESSy v2.50, but does not include the whole MESSy software package. At the moment the infrastructure modules are implemented to some extend as needed (generic submodels BLATHER, CHANNEL, CONSTANTS, SWITCH \& CONTROL, TIMER, TOOLS; cf. Jöckel et al., 2010). Additionally, the TROPOP (TROPOPause diagnostics) submodel was adapted to support ICON as base model, in order to have a simple submodel for testing and demonstration. Extensions to the MESSy code of v2.50 were necessary due to special requirements of the new base model. These changes include the implementation of the base model-specific BMIL and major changes in the generic MESSy submodels CHANNEL and TIMER, which are described in Appendix A.

\section{Hybrid parallelisation}

Shared memory parallel programming is a common technique for introducing concurrency in computer code. This parallel programming model introduces threads working concurrently on the same memory (address) space. On most present-day HPC systems, shared memory access can be used only on a "per node" base. In such environments hybrid codes can be applied, using the shared memory programming model on the nodes and a distributed memory programming model across nodes. In many applications, this is a combination of OpenMP (Open Multi-Processing; http://openmp.org/) for intra-node parallelisation and MPI (Message Passing Interface; http://www.mpi-forum.org/) for inter-node exchange.

ICON is implemented to facilitate such a hybrid parallelisation. Thus, any extension should also use this combined programming model, to yield the most efficient use of computational resources. Inter-node parallelisation is achieved, using a parallel domain decomposition, so any submodel automatically benefits. Basically, it is easy to implement intranode parallelisation, as OpenMP only requires specific directives in the code. However, it is not easy to say, where it is best to introduce this parallelisation in the submodels. Each submodel has its own computational aspects, and the gain in runtime through intra-node parallelisation critically depends on that. At this point we can only give general advice to parallelise the submodel by introducing OpenMP directives to the loops, containing the major computational work. Each submodel developer should be aware of the hybrid parallelisation concept and should test their specific developments for an efficient implementation. It is planned to introduce OpenMP parallelisation in the BMIL in a future release, to benefit from automatic parallelisation of all diagnostic submodels. This, however, includes some necessary code refactoring and a slight adaptation to the legacy submodels, which has not been started until now. In Sect. 4 we present some performance estimates for the inclusion of OpenMP parallelisation in two example submodels.

\section{GRAGG prototype}

During the $\mathrm{HD}(\mathrm{CP})^{2}$ project, we developed a prototype implementation of an online diagnostic tool, using the MESSy interface. The advanced online diagnostic tool GRAGG (GRid AGGregation) is capable of "aggregating" variables from the base model or submodels on a user-defined regular grid in different ways. User control is implemented according to the MESSy standard via Fortran namelists. 

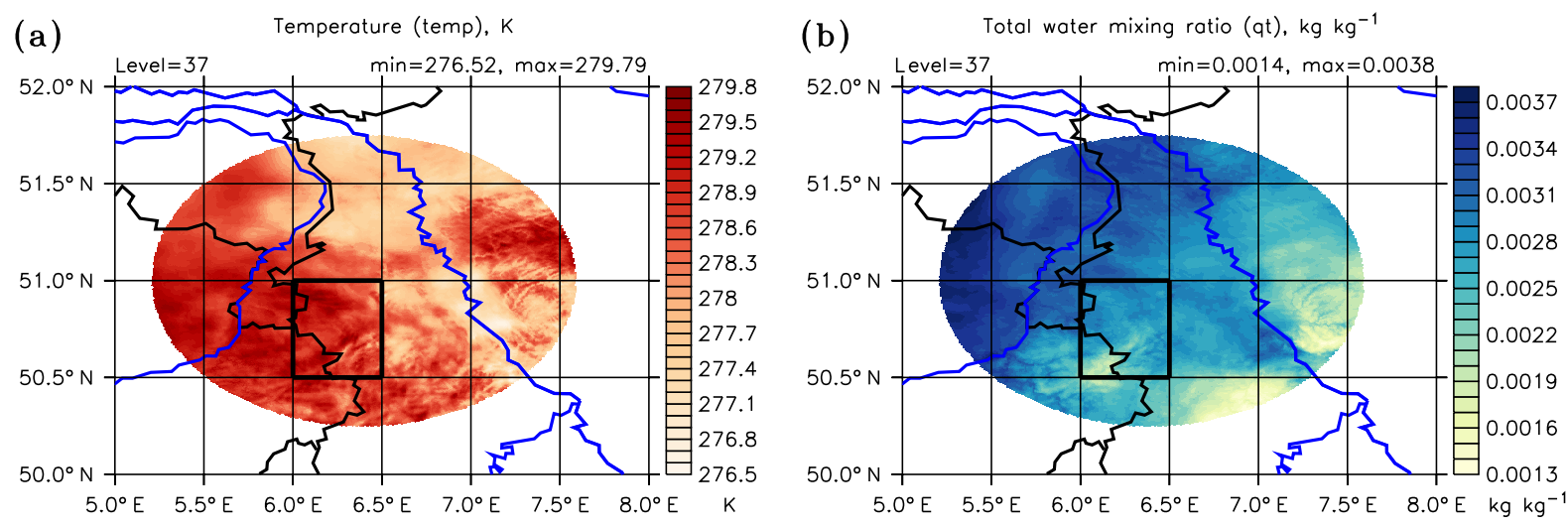

Figure 1. Snapshot on 24 April 2013, 01:00:00 UTC simulation time on model level 37 (approx. 1800 m) of the 3-D ICON model output over parts of the Netherlands, Belgium, and western Germany (a) temperature, (b) total water mixing ratio. Overlaid is the user-defined regular coarse grid $\left(0.5^{\circ} \times 0.5^{\circ}\right)$ used to perform the online diagnostics in this study. For orientation note the country borders in black and larger rivers in blue.

At the time being online operations for the spatial average, the spatial sum, the discrete (binned) probability density function (PDF), and the multivariate (for 2 variables) discrete (binned) jPDF (joint probability density function) are implemented. Furthermore, an optional area weighting can be selected by the user. For all options available via the Fortran namelist, see the GRAGG user manual in the Supplement.

Note that a temporal aggregation is not envisaged for GRAGG, because the generic CHANNEL submodel already allows time aggregation operations, such as minimum, maximum, average, and standard deviation, over the output time interval (see Sect. 2 and the Supplement of Jöckel et al., 2010). This facility can also be used for the variables calculated in GRAGG.

\subsection{Implementation details of the prototype}

The diagnostic submodel GRAGG operates in two phases, the initialisation phase and the time integration loop. During initialisation, the submodel processes the user namelist and initialises the memory. GRAGG stores, for each cell on the coarse user-defined regular grid, triangle indices from the native ICON grid, accessible in the physical memory of the respective processing entity (PE; corresponding to MPI task in this case). Simultaneously it counts the number of triangles present on the PE and their area in each coarse grid box. These values are communicated to the other PEs and combined to the total number of triangles and their total area per grid box. Weighting coefficients are calculated for each triangle from its respective area and the total area in the coarse grid box.

During the time integration loop, partial results are calculated on each PE. For summation and spatial averaging this is the sum over all values of the local triangles per grid box, with subsequent normalisation with the total number of triangles in each box for the averaging operation. To achieve an efficient storage of jPDFs, GRAGG determines minimum and maximum of the distribution from the variables in every time step per coarse grid box. This requires inter-task communication, as the memory locations of triangles contained in one box may be distributed over multiple MPI tasks.

For the (j)PDF, the bin width is determined from the userdefined number of bins and the calculated minimum and maximum in the coarse grid box. In a loop over all triangles in the grid box, GRAGG determines in which bin the value has to be put. The number of values sorted into the bins are counted in a "bin vector", which holds the partial result of the (j)PDF for each grid box. After the loop over all triangles on the PE, the bin vectors for all grid boxes are normalised by division of the total number of triangles in the respective box. More details on the (j)PDF calculation can be found in the GRAGG user manual, which is part of the Supplement.

The last step takes place immediately before the output. Now the results from the partial calculation on the processes are collected and summed up to form the overall result in the output fields. The advantage of splitting the calculation in determining partial results on the processes during the time loop and the final combination to a global result in the output time step is the minimisation of inter-process communication during the time loop. However, we have to keep in mind, that during the time loop only partial results on the PEs are available.

\subsection{Evaluation of GRAGG}

To show the capabilities of the prototype GRAGG, we conducted a short ICON simulation with the diagnostic interface and the GRAGG submodel activated. Figure 1 shows ICON model output snapshots of the innermost domain over parts of the Netherlands, Belgium, and western Germany on 24 April 2013, 01:00:00 UTC simulation time on model level 37 (approx. $1800 \mathrm{~m}$ ) for temperature and total water mixing 


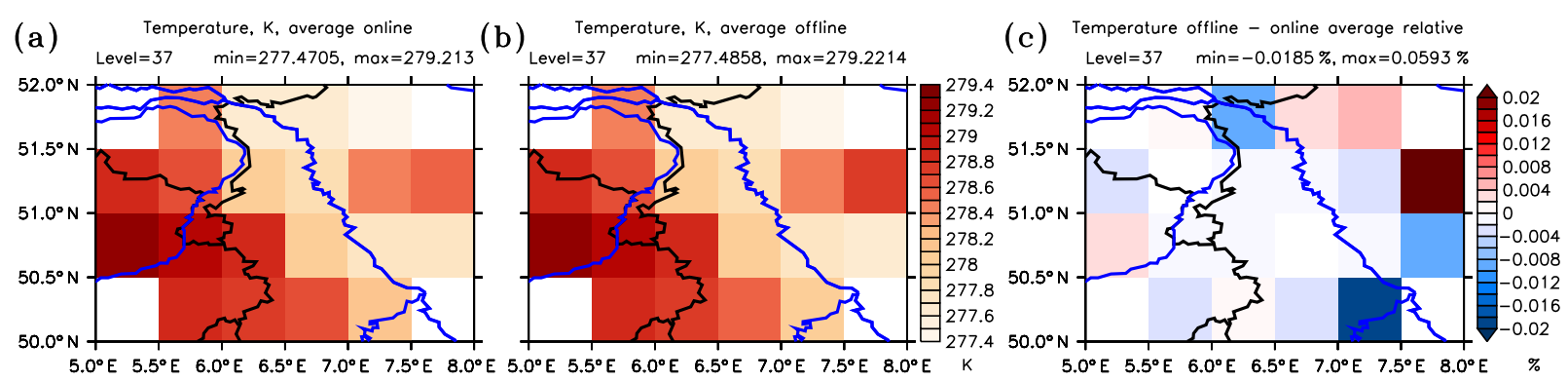

Figure 2. Temperature on 24 April 2013, 01:00:00 UTC simulation time on model level 37 (approx. $1800 \mathrm{~m}$ ) averaged on the $0.5^{\circ} \times 0.5^{\circ}$ grid. (a) Output of online calculation from GRAGG, (b) offline calculation from 3-D ICON model output, (c) relative difference between offline and online calculated values. For orientation note the country borders in black and larger rivers in blue.

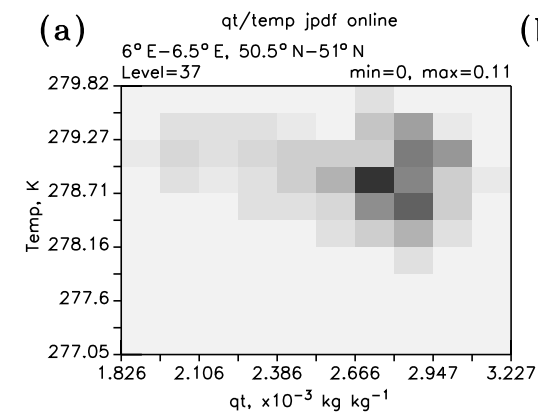

(b) at/temp jpdf offline

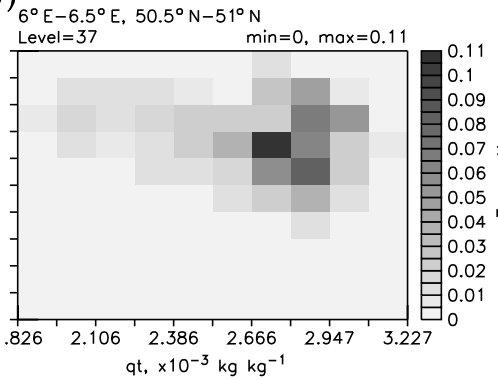

(c) at/temp jpdf offline - online relotive

\begin{tabular}{l}
$6^{\circ} \mathrm{E}-6.5^{\circ} \mathrm{E}, 50.5^{\circ} \mathrm{N}-51^{\circ} \mathrm{N}$ \\
279.82 Level $=37 \quad \min =-0.37 \%, \max =0.14 \%$ \\
\hline
\end{tabular}

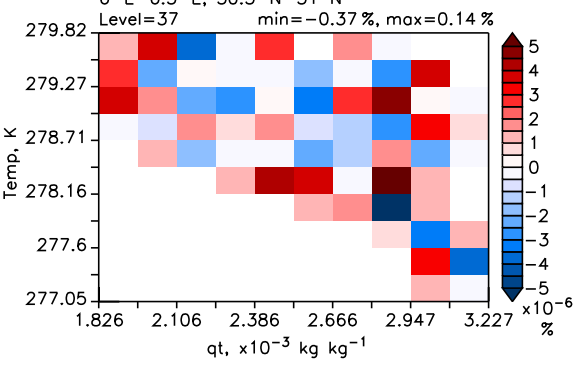

Figure 3. Joint PDF of water mixing ratio and temperature on 24 April 2013, 01:00:00 UTC simulation time on model level 37 (approx. $1800 \mathrm{~m}$ ) in grid box $6.0-6.5^{\circ} \mathrm{E}, 50.5-51.0^{\circ} \mathrm{N}$. (a) Output of online calculation from GRAGG, (b) offline calculation from 3-D ICON model output, (c) relative difference between offline and online calculated values.

ratio. The grid spacing of the innermost domain of the simulation is $312 \mathrm{~m}$. GRAGG was set up to calculate the spatial average of temperature and the jPDF of total water mixing ratio and temperature on a user-defined $0.5^{\circ} \times 0.5^{\circ}$ grid. We compare the results from GRAGG with offline calculations based on the three-dimensional (3-D) ICON output; the Ferret (http://ferret.pmel.noaa.gov/Ferret) scripts used to process the model data are available in the Supplement.

An example of the temperature averaged on the userdefined regular coarse grid on model level 37 (corresponding to approx. $1800 \mathrm{~m}$ ) is shown in Fig. 2. The online calculated average temperature from the diagnostic tool is shown on the left (Fig. 2a), the same aggregation operation calculated from 3-D ICON output offline in the middle (Fig. 2b). The relative differences between the direct GRAGG output and the offline calculation are lower than $6 \times 10^{-2} \%$ (Fig. $2 \mathrm{c}$ ).

The jPDF of total water mixing ratio and temperature in the highlighted box of Fig. 1 on model level 37 is shown in Fig. $3 a$ and $b$ for the online GRAGG output and the offline calculation, respectively. As we see from Fig. 3, the relative differences mostly range between $-5 \times 10^{-6}$ and $5 \times 10^{-6} \%$, with one bin reaching $-0.37 \%$ and one bin reaching $0.14 \%$.

The discrepancies can be explained, as the offline calculation operates on a single-precision output, whereas the online diagnostic makes use of the full double-precision values available during the model simulation. The examples shown here demonstrate the ability to get the same results from the online diagnostic as for the post-processing of model data.

\section{Application of online diagnostics}

In the following application, we use GRAGG to retrieve jPDFs of total water and temperature. As outlined in Sect. 3.1, GRAGG uses demanding communication patterns during the calculation of jPDFs. Thus, this task could be seen as one extreme with respect to the usage of computer resources. In this study we will use this submodel as example of a "communication bound" online diagnostic tool.

As an example for a submodel with low inter-task communication, we will use VISOP (VIsual Satellite OPerator), developed at the Ludwig-Maximilians-Universität (LMU) during the $\mathrm{HD}(\mathrm{CP})^{2}$ project (Scheck et al., 2016). This submodel implements a radiation calculation along model columns, i.e. stacks of triangles on the ICON grid. The implementation is column-based and inter-task communication only happens during output time steps of the MESSy interface. This communication is necessary at the moment, because the interface performs only serial I/O (cf. Sect. 3.1). VISOP is an example of a "calculation bound" submodel, as most of the work in the time step is spent in calculations over the model columns. 
Table 1. Overview of the test cases for the performance tests.

\begin{tabular}{|c|c|c|c|c|c|}
\hline \multirow[b]{2}{*}{ Name } & \multirow[b]{2}{*}{$\begin{array}{l}\text { MESSy } \\
\text { interface }\end{array}$} & \multicolumn{2}{|c|}{ Submodels } & \multicolumn{2}{|c|}{ Optimisation } \\
\hline & & GRAGG & VISOP & OpenMP & $\begin{array}{c}\text { reduced } \\
\text { call frequency }\end{array}$ \\
\hline ICON only & & & & $\mathrm{a}$ & \\
\hline MESSy interface & $x$ & & & & \\
\hline MESSy + GRAGG & $x$ & $x$ & & & \\
\hline MESSy + GRAGG OMP & $x$ & $x$ & & $x$ & \\
\hline MESSy + GRAGG reduced & $x$ & $x$ & & & $x$ \\
\hline MESSy + GRAGG red. + OMP & $x$ & $x$ & & $x$ & $x$ \\
\hline MESSy + VISOP & $x$ & & $x$ & & \\
\hline MESSy + VISOP OMP & $x$ & & $x$ & $x$ & \\
\hline MESSy + VISOP reduced & $x$ & & $x$ & & $x$ \\
\hline MESSy + VISOP red. + OMP & $x$ & & $x$ & $x$ & $x$ \\
\hline
\end{tabular}

${ }^{a}$ OpenMP in the base model ICON is always enabled. ${ }^{b}$ With OpenMP parallelisation.

\subsection{Model set-up}

We performed several runtime tests on Hochleistungsrechnersystem für die Erdsystemforschung 3 (HLRE-3, HPC system for Earth System research 3) at DKRZ to test the performance of the MESSy interface in ICON itself and the performance of the different submodels. We switched on or off specific components of the code to quantify the influence of the respective component on the model runtime. Table 1 lists all test cases and their specific set-ups. The tests consisted of simulations with the MESSy interface included and not included in the code, with the submodels GRAGG and VISOP switched on or off, with code modifications in the submodels to reduce the call frequency of calculations in the submodel to the output time step only, and with OpenMP parallelisation of the loop(s) containing the main work of the submodel. A reduction of the call frequency can be only applied if the calculations in the submodel do not rely on any data from other time steps than the current one, and if calculated data are not required by other submodels.

For all tests we used revision r25413 from 16 November 2015 of the development repository icon-diag-hdcp2-refactor (https://svn. zmaw.de/svn/icon/branches/icon-diag-hdcp2-refactor).

The code was compiled with Intel Fortran Version 14.0.3.174 Build 20140422 and linked against bullx MPI version 1.2.8.3 and netCDF 4.4.2 libraries. We configured the code with./configure --with-fortran=intel --with-openmp

--with-mpi=/opt/mpi/bullxmpi_mlx/1.2.8.3 --with-flags=hiopt, for the inclusion of the MESSy interface we added --with-messy. This results in compiler flags (include and preprocessor flags dropped): -openmp -03-mkl=sequential -pc64 -no-prec-div -no-prec-sqrt

-fast-transcendentals -reentrancy

threaded -xHost -vec-report 1.
The set-up of all test cases was based on the experiment exp.ICOLES_nestgrid. It includes three nested domains over Germany, with grid spacing of 1249,625 , and $312 \mathrm{~m}$ and 302912, 893548 , and 224132 grid cells, respectively, resulting in 1420592 horizontal grid cells in total. Each nested domain has 50 vertical levels. The large eddy simulation (LES) physics package with a two moment microphysics scheme is used (Dipankar et al., 2015). The simulations were integrated over $1 \mathrm{~h}$ simulation time, starting 24 April 2013, 00:00 UTC, with an integration time step of $10 \mathrm{~s}$. For all simulations we used 4 MPI tasks per node and 6 OpenMP threads per task. Asynchronous output of the ICON model was carried out by six processors.

When activated, the standard ICON output consisted of 35 meteogram stations with an output interval of 50 time steps, the output of 13 model variables (2-D) on the innermost domain every $15 \mathrm{~min}$, and 20 cloud-related diagnostic variables (2-D) on all domains every $30 \mathrm{~min}$. Output of variables related to the planetary boundary and the land model (32 2-D and $23-D$ variables, and 3 variables on 9 or 8 vertical levels) occurred every hour. Prognostic and radiation variables, and physical tendencies (altogether 24 3-D and 27 2-D variables) were written out every $3 \mathrm{~h}$. The output of VISOP contained 10 2-D variables, the output of GRAGG as used in the test cases consisted of 6 variables on every model level on a reduced regular grid of $0.1^{\circ} \times 0.1^{\circ}(121 \times 81$ grid points $)$, and one jPDF (3-D on the reduced grid times 100 bins). In this study we used an output interval of $15 \mathrm{~min}$ for the diagnostic submodels.

\subsection{Performance tests}

For the analysis of model runtime, we utilised the timer debug mechanism already included in ICON. The results discussed here are the values reported for timer total. Tables with all runtimes can be found in the Supplement. In the following we use the term overhead $O_{\text {test case,base case }}(n)$ of a test 


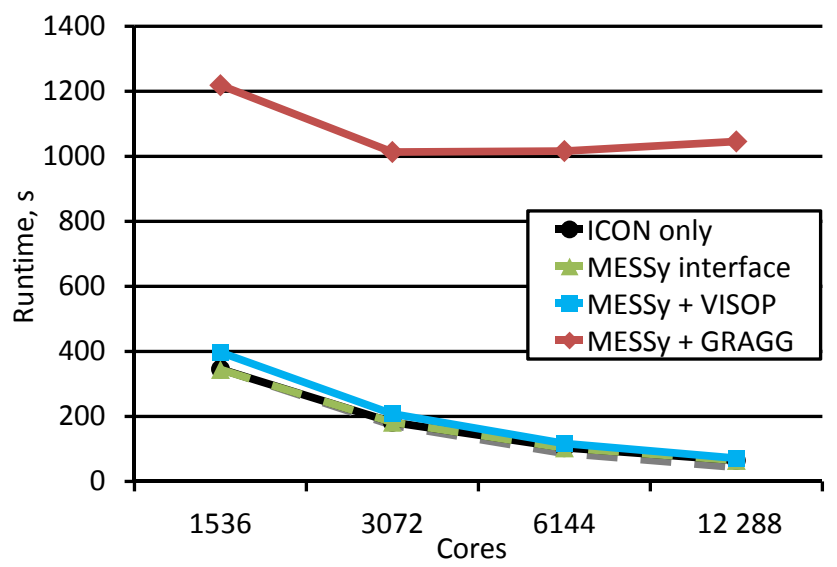

Figure 4. Runtime (s) for different test cases with deactivated output, measured with the internal timer "total" on $64,128,256$, and 512 nodes (1536, 3072, 6144, and 12288 cores, respectively) of HLRE-3. The dashed grey line marks perfect scaling for "ICON only".

case relative to a base case simulation, defined as

$O_{\text {test case, base case }}(n)=\frac{t_{\text {test case }}(n)}{t_{\text {base case }}(n)}-1$,

with $t_{\text {test case }}(n)$ the runtime of a test case on $n$ nodes, and $t_{\text {base case }}(n)$ the runtime of a base case on the same number of nodes. In this study "ICON only" with or without output is considered as the respective base case, in order to calculate the additional fraction of runtime caused by the diagnostic interface and the diagnostic tools.

The speed-up $S_{\text {test case }}(n)$ is calculated for each test case simulation from the model runtimes $t_{\text {test case }}$ on $n$ nodes relative to the corresponding simulation on 64 nodes:

$S_{\text {test case }}(n)=\frac{t_{\text {test case }}(n=64)}{t_{\text {test case }}(n)}$.

Tables with calculated speed-ups for all test cases can be found in the Supplement.

The extension of ICON through the compilation of the model system with the MESSy interface does not introduce any significant overhead in terms of runtime of the model system (Figs. 4, 5). All increases in runtime due to the activation of the MESSy interface are below $0.1 \%$. Measurable overhead is only introduced by activating diagnostic tools.

The scaling behaviour of the two diagnostic tools tested here with an increasing number of computer cores differ significantly. Whereas the overhead increases with higher number of cores for GRAGG, it decreases for VISOP. In both cases this behaviour is very similar regardless of activated output. From these results we can see the different nature of the two submodels. Whereas GRAGG is bound by intertask communication, which increases the overhead with additional tasks, VISOP is bound by its calculations, which results in a better scaling with additional tasks (at least up to the number of 12288 cores, tested here).

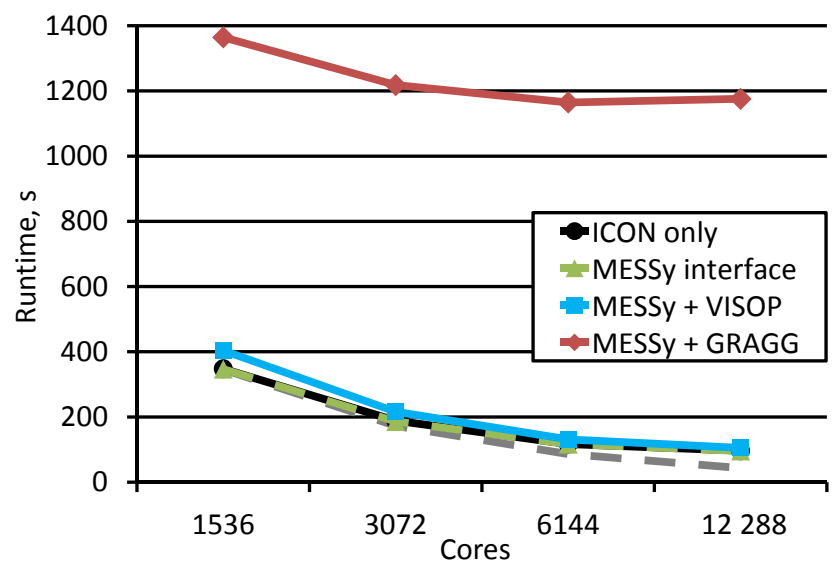

Figure 5. As Fig. 4 with output activated for ICON and the submodels.

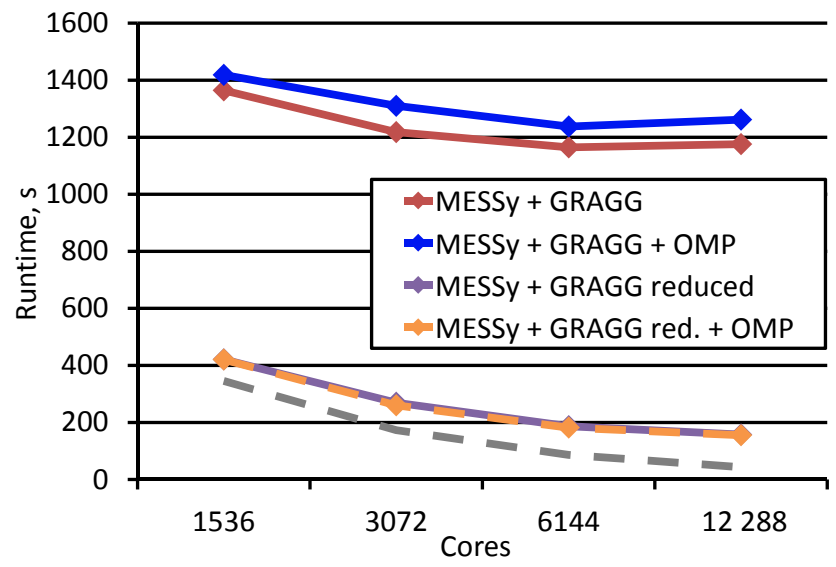

Figure 6. Comparison of the runtime (s) of GRAGG and versions of GRAGG with reduced call frequency, OpenMP, and a combination of both. Output was activated in all test cases.

To optimise the runtime behaviour of the two submodels, we explored the possibility of making efficient use of the hybrid parallelisation approach by introducing OpenMP regions. Furthermore, to reduce the overall runtime of the model, we studied the effect of a reduced call frequency of the submodels. For this, we reduced the call frequency of the submodels to the output time step of the corresponding submodel (15 min).

The comparison of the different optimisations for GRAGG and VISOP are shown in Figs. 6 and 7, respectively. In Fig. 8 the comparison of both test cases with the lowest runtime for the submodels GRAGG and VISOP, and the "ICON only" base case is shown.

As the bulk in runtime of GRAGG results from MPI communication tasks, GRAGG does not benefit from OpenMP parallelisation of its calculation loops (Fig. 6). The total runtime even increases, due to the additional but inevitable overhead introduced by OpenMP. The additional MPI communication for the determination of minimum and maximum 


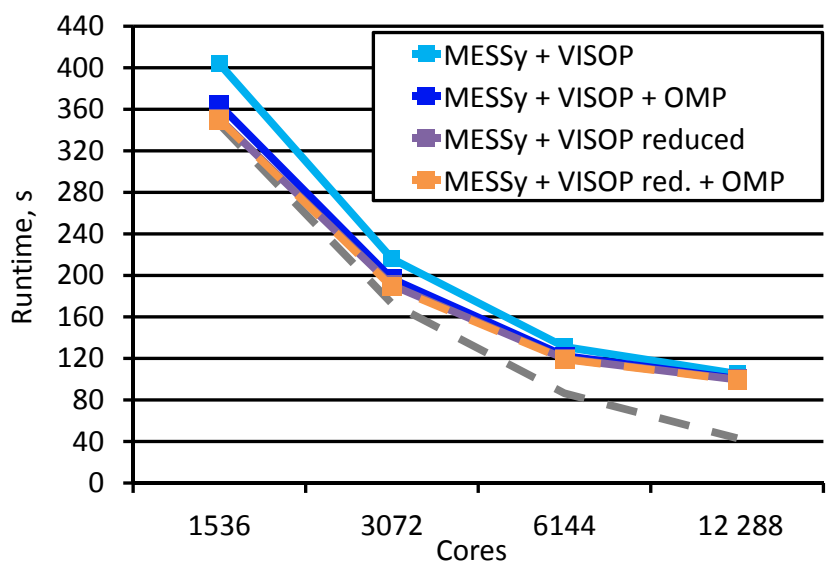

Figure 7. Comparison of the runtime (s) of VISOP and versions of VISOP with reduced call frequency, OpenMP, and a combination of both. Output was activated in all test cases.

causes bulk of the additional runtime (cf. Sect. 3.1). The only applicable strategy to reduce the runtime of GRAGG is to restrict calls of the submodel to output time steps only. This reduces the overhead from GRAGG compared to "ICON only" (no output) to 2.6 and $19.37 \%$ for 64 and 512 nodes, respectively. Between the versions of GRAGG with and without OpenMP parallelisation only slight differences in the overhead exist. In the case we activate the output, overheads for this optimised version of GRAGG increase to 20.86 and $60.79 \%$, respectively. The reduction of the call frequency of GRAGG yields a better scalability of the model system, resulting in a higher speed-up (Fig. 9). For GRAGG with reduced call frequency and output, the speed-up on 512 nodes is 2.71 and 2.67, with and without OpenMP, respectively. The values for the test case without output are 4.58 and 4.59 , respectively. GRAGG operated on the full temporal resolution only reaches speed-up values slightly more than 1 (Fig. 9). This shows a bad scaling behaviour for this MPI communication intensive tool.

For VISOP we see the benefit of parallelising the submodel using OpenMP (Fig. 7). This significantly reduces the overhead for VISOP by introducing simple OpenMP directives for the main calculation loop. Without OpenMP, the lowest overhead from VISOP is reached for 512 nodes, achieving values of 9.13 and $10.6 \%$ with and without output, respectively. Overall, with a reduced call frequency and OpenMP parallelisation we can reduce the overhead of VISOP to $2.72 \%$ (for 512 nodes and activated output) and lower. Thus, with the optimised version of VISOP the total model runtime of $99.08 \mathrm{~s}$ (without initialisation) is only increased by $2.67 \mathrm{~s}$.

The speed-up for VISOP on 512 nodes is more than 5 without output and reaches more than 3.5 with output in all cases (Fig. 9). These values are above the speed-ups for the test cases with GRAGG. A reduced scalability because of the limited I/O capabilities of the diagnostic interface (cf.

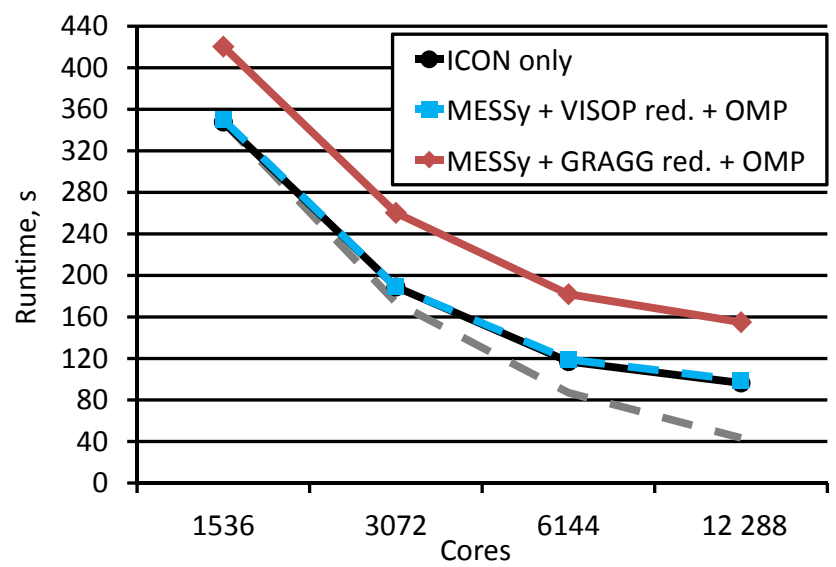

Figure 8. Comparison of the runtime (s) of "ICON only", and the test cases with the lowest runtime for activated GRAGG and VISOP. Output was activated in all test cases. The dashed grey line marks perfect scaling for "ICON only" with activated output.

Sect. 2.3) is obvious. But all speed-ups are comparable to the values obtained from the test cases of the ICON model without the diagnostic interface. The worse scaling for GRAGG compared to VISOP in all test cases with diagnostic output is caused by the larger amount of data written out and the additional MPI communication during each output step. The bad scaling behaviour of GRAGG operating on the full temporal resolution is caused by the intensive inter-task communication during each time step (cf. Sect. 3.1).

The initialisation of the submodels takes only a few percent of the time the total model system needs for initialisation. We do not present any absolute values here, as there is a large variability in the times measured for initialisation. The initialisation includes extensive $\mathrm{I} / \mathrm{O}$ operations for reading in the model grid and the domain decomposition, and hence the time for initialisation is influenced by $\mathrm{I} / \mathrm{O}$ system usage of other applications on the HPC system.

With all configurations we reach an acceptable speed-up, which obviously is reduced in test cases with activated output (Fig. 9). This is related to the I/O bottleneck and how I/O operations are currently implemented in the diagnostic tools. Whereas ICON uses an asynchronous output strategy, which enables execution of $\mathrm{I} / \mathrm{O}$ (partly) in parallel to the model calculations, the output from submodels using the MESSy infrastructure is at the moment strictly serial. The runtime of the test cases with optimised code of VISOP and GRAGG including output are shown in Fig. 8. We find a very low overhead for VISOP. The additional runtime for GRAGG ranges between 58.6 and $72.56 \mathrm{~s}$ for the fully optimised test case with output. Compared to the simulations with the optimised GRAGG without output, 79.8 to $89.5 \%$ of the additional runtime ( 512 nodes and 64 nodes, respectively) is caused by the activation of output. To increase the speed-up and reduce the overall time to solution, efficient and parallelised output is a critical topic that we are working on. 


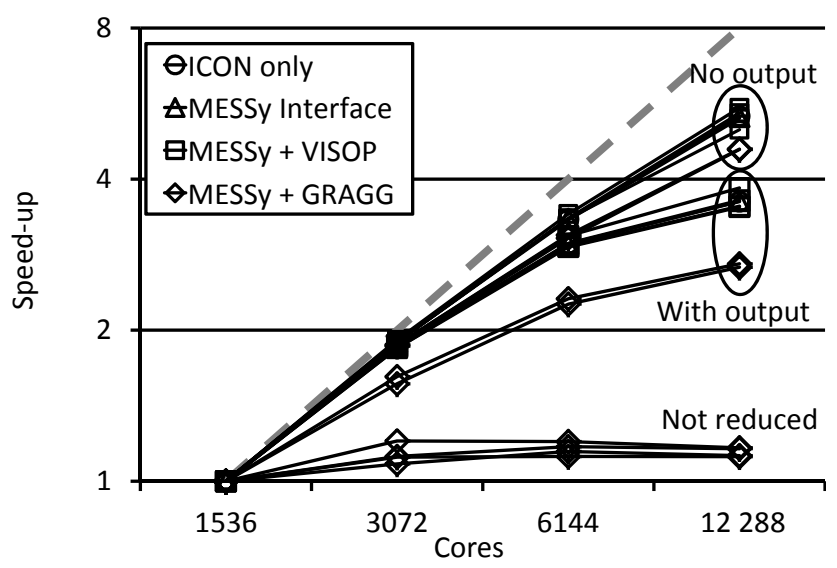

Figure 9. Speed-up calculated to the baseline of 64 nodes (1536 cores) for each test case. Symbols match those in Figs. 4-7. Labels mark simulations with and without output. For "MESSy + GRAGG", the lower four series are speed-ups for simulations with GRAGG operating on the full temporal resolution. The dashed grey line represents optimal speed-up.

For the MESSy interface and the diagnostic tool VISOP, the memory consumption of the extension compared to the ICON model itself is modest (Fig. 10). Generally, the memory consumption per node is larger for the test cases with output activated. Simulations with GRAGG have larger memory demands compared to the simulations with VISOP or without any submodel. This is due to the higher buffering demands of the increased MPI communication and internally used (temporal) fields. The largest memory consumption is reached for the simulations with GRAGG and activated output.

In general the memory demands differ substantially, depending on how much internal and output variables the submodels need. We are further investigating the memory demands of the model system. We want to reach a memory footprint that is as small as possible, especially facing the actually decreasing ratio of memory per compute core on HPC systems. This should also be kept in mind when developing novel diagnostic tools to be run online in the model system.

\section{Ongoing and future developments}

At the moment we are porting our developments of the diagnostic interface and the diagnostic tools to the Jülich Blue Gene/Q HPC system JUQUEEN. First tests on this IBM PowerPC architecture are running successfully, but at the moment these tests are constrained to a fixed combination of 4 MPI tasks per node in combination with 4 OpenMP threads per task. The biggest challenge on that system is the low amount of available memory per core of $1 \mathrm{GiB}$, so we have to optimise our implementation towards a minimum memory footprint.

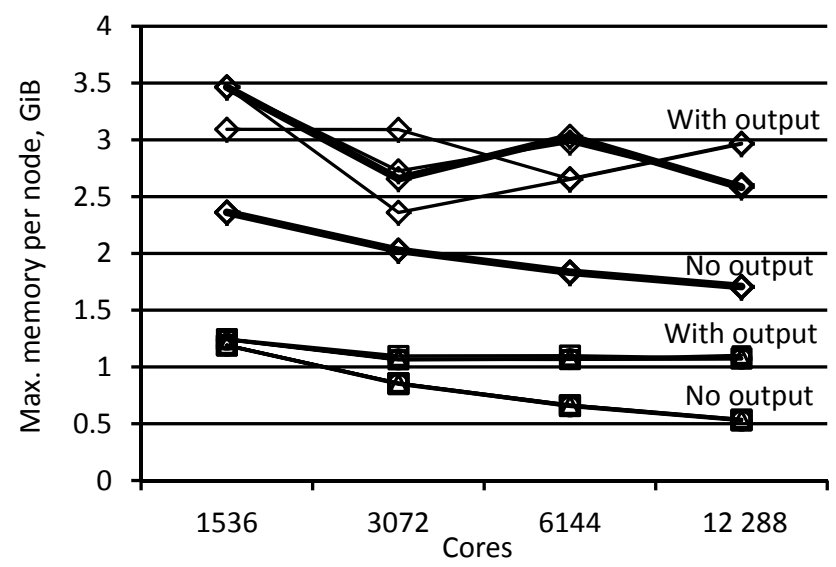

Figure 10. Maximum of memory consumption per node. Symbols as in Fig. 9.

Furthermore, more diagnostic submodels are being developed during $\mathrm{HD}(\mathrm{CP})^{2}$ in the areas of satellite simulators (COSP, Cloud Feedback Model Intercomparison Project's Observation Simulator Package; Bodas-Salcedo et al., 2008) and online trajectories. Our future plans include the implementation of an online feature identification and tracking system, and further forward operators, for improved comparison between model simulations and measurements.

We are working on further optimisation of the diagnostic interface and tools together with scientists of DKRZ in the second phase of $\mathrm{HD}(\mathrm{CP})^{2}$. This includes the integration of parallel output in the interface, as soon as the improved parallel version of CDI is available in ICON.

Last, but not least, as there is already a re-gridding tool available in the MESSy framework, an integration into the GRID submodel (Kerkweg and Jöckel, 2015) with further generalisation of the aggregation developed in this study seems beneficial.

\section{Conclusions}

We presented the implementation of parts of the Modular Earth Submodel System (MESSy) into the ICOsahedral Nonhydrostatic (ICON) model framework for the application as diagnostic interface. This defines a generalised and easyto-use interface for the implementation of online diagnostic tools. Because of the generalisation, the inclusion of the interface into other numerical models provides the possibility to operate the same diagnostic tools. This enhances the consistency of output between different models and reduces the need for re-implementation of already existing and working code.

We will be able to reduce $\mathrm{I} / \mathrm{O}$ operations during future model simulations, as we write out online aggregated variables and reduce the need of storing high-resolution 3-D fields of model variables. Furthermore, as we reduce the need 
for (offline) post-processing to derive diagnostic variables, we can reduce further sub-sequent access to the I/O system. However, the use of online diagnostic tools during the model simulations increases the runtime of the simulation, but we have to keep in mind, that post-processing tools do not come without computational costs.

There is a trade-off between the time needed for additional computations and the time needed for access to the I/O system. On the one hand, we could stay with the computational demands of the model system, the post-processing tools and their access to the I/O system. On the other hand, we could increase computational demands for a simulation, while reducing the demand for post-processing and the overall access to the I/O system. When the simulation is highly data intensive, which is the case for high-resolution simulations of ESMs, there is a chance to reduce the "time-to solution" on systems with an I/O bottleneck. The increase in runtime of the model simulation due to additional online diagnostics can be compensated by the omission of post-processing and by lower time spent for I/O operations.

We showed that the integration of the diagnostic interface itself has no impact on the model system runtime and memory consumption. The overhead introduced by diagnostic submodels, however, can be substantial, but could be reduced by thorough code optimisation. We reached a maximum overhead of $60.79 \%$ for the "communication bound" example of the jPDF diagnostic in GRAGG and a maximum overhead of $2.72 \%$ for the "computational bound" example of the satellite operator VISOP. Both simulations applied a reduced call frequency of the diagnostic tools and included output. Our future work will focus on the optimisation of the remaining I/O operations and the optimisation of the memory footprint. Furthermore, we will support the development efforts of additional online diagnostics utilising the MESSy interface.

The ratio between I/O bandwidth and peak performance of high-performance systems decreased in the recent years, and probably will continue to do so for future systems, as the performance gap widens. Additionally, the increase in storage capacity has slowed down recently. We have to focus on these problems during the design and provision phase of future HPC systems. Apparently, there will be no quick solutions to the problems, as the widening of the performance gap might persist for decades. For now, we will have to focus on software solutions to bridge this gap by providing efficient I/O operations, making use of parallelisation and appropriate I/O middleware in our codes, and also by reducing the amount of output data. Recent studies focus on data compression as a (transparent) post-processing step, either when the data are stored, or even online before data are transferred over the computer network to increase communication performance.
We suggest the application of online diagnostic tools to reduce the volume of data from numerical simulations. This will require a largely modified workflow for scientists, as appropriate analyses have to be selected and online tools have to be developed before the simulation starts.

Checkpointing is already applied by model systems to overcome restrictions in compute times of job schedulers, and to restore simulations in case of software or hardware failures. Even when cutting the "full" model output to a minimum, a subsequent re-calculation with increased output volume and frequency is therefore possible. Thus, scientists should not be afraid of "losing" data, as a combination of all methods should suit their needs.

Currently applied workflows may not retain the same on future HPC systems. A certain degree of development and optimisation of current code and the application of novel methods are required. Here, a generalised interface for online diagnostic tools is extremely useful.

\section{Code availability}

The Modular Earth Submodel System (MESSy) is continuously further developed and applied by a consortium of institutions. The usage of MESSy and access to the source code is licensed to all affiliates of institutions that are members of the MESSy Consortium. Institutions can be a member of the MESSy Consortium by signing the MESSy Memorandum of Understanding. More information can be found on the MESSy Consortium website (http://www.messy-interface. org). The MESSy infrastructure submodels are freely available from the authors.

ICON (ICON atmosphere) will be made available under the ICON Software License Agreement ISLA version2.1, which will be a common SLA of the German Weather Service DWD and the MPI-M. Additional details for licensing ICON can be found at http://www.mpimet.mpg.de/en/ science/models/license/.

For model developers, who obtained the proper licenses, a development version of the code is available from the authentication restricted repository at https://svn.zmaw.de/svn/ icon/branches/icon-diag-interface. 


\section{Appendix A: MESSy-specific code extensions}

The generic MESSy submodel CHANNEL (Jöckel et al., 2010) implements an interface for exchange of data between the base model and submodels, and among submodels, and handles the export of data to files. It is implemented in an object-oriented way and offers extended control of data flow, e.g. an enhanced user-defined output control.

Time management is controlled via the generic submodel TIMER, which is based on the timer of the atmospheric general circulation model (GCM) ECHAM5. For the reimplementation as generic MESSy submodel, enabling its application for various base models, the timer relevant code was extracted from the GCM, keeping its original functionality and namelist syntax (Jöckel et al., 2010).

In the following sections, we present extensions to the development cycle 2 of the MESSy interface and its generic submodels CHANNEL and TIMER (Jöckel et al., 2010), resulting from special requirements of ICON as base model. More details on the MESSy interface and the implementation of submodels can be found in Jöckel et al. (2005, 2010). An extended version of the user manual for CHANNEL is included in the Supplement of the present paper.

\section{A1 CHANNEL}

The generic MESSy submodel CHANNEL manages data objects in a hierarchical structure build-up from channels and channel objects. Details can be found in Jöckel et al. (2010) and the CHANNEL user manual available from the Supplement.

ICON provides the capability to be operated with refined nests, called domains or patches. One-way or two-way nesting can be used in the global configuration of the model as well as in the limited area mode. When a MESSy BMIL subroutine is called, the patch on which the base model is operating at that moment is passed to the subroutine by a parameter patch_id. The information is available to MESSy submodels via the variable current_patch (see below).

Although nesting of refined domains is supported in MESSy (Kerkweg and Jöckel, 2012b), this is only available for direct external coupling (cf. Appendix A of Kerkweg and Jöckel, 2012b), which utilises different program units for the different nests. In this configuration CHANNEL has not to be aware of different nests, as the nested domains reside in different namespaces, and are separated physically in memory and storage. As refined nested patches in ICON use internal direct coupling in one executable, we had to extend CHANNEL to support multiple patches for data management and storage. Changes in the application programming interface (API) are implemented using optional subroutine parameters, ensuring full backward compatibility for existing submodels. Here, we describe the structural changes of the CHANNEL source code and the corresponding changes in the API of CHANNEL subroutines in brief.
The complete list of channels is stored in a concatenated list. Until now, one pointer GCHANNELLIST is defined in smcl/messy_main_channel.f 90 pointing at the first element of the channel list. To support more than one patch, we introduced a wrapper t_channel_list_ptr for the channel list, which allows for allocating as many channel_list_ptr as there are patches in a simulation. In the standard case, when the base model does not allow native nesting (or no nests are defined), only one channel_list_ptr is allocated. This allows for backward compatibility by applying all operations on channels and channel objects only to the elements of the first (and only) channel list in that case.

To avoid the need of explicitly passing the patch on which the base model is currently operating to every subroutine, a public variable current_patch was introduced to the CHANNEL source code (in smcl/messy_main_channel.f90). This variable is set in the BMIL and can be accessed by any submodel via the Fortran USE. In CHANNEL subroutines the optional parameter patch_id controls, which patch is accessed. Access of channels and channel objects from subroutines unaware of multiple patches is transparent, as in calls without the patch_id set, the subroutine works on the first patch. This allows for full backward compatibility of legacy submodels designed to work with base models without support of native nesting.

The concept of representations and dimensions as basic entities of the CHANNEL submodel is still valid in the implementation for ICON. As sizes of dimensions in different patches usually differ, these entities are stored separately for each patch. This concept allows one to use identical names on the different patches, while additionally providing the patch_id when accessing these entities. This is transparent for the user of CHANNEL, but the submodel developer has to take care, if the submodel uses representation or dimension ID-numbers directly. In this case, the patch_id has to be used as index to the list of the representation or dimension ID-numbers.

\section{A2 TIMER}

Until now, MESSy uses the generic TIMER submodel, reimplemented from the extracted time management code of the GCM ECHAM5 (Roeckner et al., 2006). It uses seconds as base unit and is synchronised to the base model timer each time step. For high-resolution simulations using a grid spacing in the order of $100 \mathrm{~m}-$ as it is planned for the $\operatorname{HD}(C P)^{2}$ simulations - a smaller granularity in time management is needed. Our extension provides a granularity of milliseconds. Future plans include the change of the MESSy time management to an external library (MTIME), to be consistent with the ICON time management and to make use of its enhanced capabilities. 


\section{A3 Submodels}

All extensions to MESSy are implemented in a way to ensure backward compatibility. Thus, only minor updates to the SMIL modules of existing submodels are necessary.

The major modification was introduced, because we need to provide the ability of submodels to work on all patches defined by the base model. For this, a standardised subroutine $<$ submodel>_set_pointer is called every time the simulation continues on a different patch. The purpose of this subroutine is to set the local pointers of the submodel to the appropriate memory in the current patch of the base model. 


\section{Appendix B: Glossary}

\begin{tabular}{|c|c|}
\hline API & Application programming interface \\
\hline Base model & $\begin{array}{l}\text { A general circulation model, numerical weather model, or box model extended by calls to MESSy } \\
\text { specific routines }\end{array}$ \\
\hline BMBF & German Federal Ministry of Education and Research (Bundesministerium für Bildung und Forschung) \\
\hline BMIL & Base model interface layer \\
\hline BML & Base model layer \\
\hline Channel & A set of channel objects and additional meta-information in the CHANNEL submodel \\
\hline Channel list & In this structure, all channels are stored \\
\hline Channel object & Data and its meta-information \\
\hline CLaMS & Chemical Lagrangian Model of the Stratosphere \\
\hline CDI & Climate Data Interface \\
\hline CCSM & Community Climate System Model \\
\hline CESM & Community Earth System Model \\
\hline COSMO & Consortium for Small-scale Modelling model \\
\hline COSP & Cloud Feedback Model Intercomparison Project's Observation Simulator Package \\
\hline Dimension & Describes the structure of data in a particular spatial or temporal direction \\
\hline DKRZ & German Climate Computing Centre (Deutsches Klimarechenzentrum) \\
\hline Domain & Spatial extend on which the model operates, different domains can be nested \\
\hline DWD & German Weather Service (Deutscher Wetterdienst) \\
\hline ECHAM5 & European Centre HAMburg general circulation model, version 5 \\
\hline EMAC & ECHAM5/MESSy Atmospheric Chemistry model \\
\hline ESM & Earth system model \\
\hline ESMF & Earth System Modeling Framework \\
\hline GCM & General circulation model \\
\hline GRAGG & GRid AGGregation submodel \\
\hline $\mathrm{HD}(\mathrm{CP})^{2}$ & High Definition Clouds and Precipitation for advancing Climate Prediction \\
\hline HLRE-3 & HPC system for Earth System research 3 (Hochleistungsrechnersystem für die Erdsystemforschung 3) \\
\hline $\mathrm{HPC}$ & High-performance computing \\
\hline ICON & ICOsahedral Non-hydrostatic model system \\
\hline $\mathrm{I} / \mathrm{O}$ & Input and Output \\
\hline jPDF & Joint probability density function \\
\hline LES & Large eddy simulation \\
\hline LMU & Ludwig-Maximilians-Universität, Munich \\
\hline MESSy & Modular Earth Submodel System \\
\hline MPI-M & Max Planck Institute for Meteorology \\
\hline MPI & Message Passing Interface \\
\hline OpenMP & Open Multi-Processing \\
\hline Patch & Spatial extend on which the model operates, different patches can be nested \\
\hline $\mathrm{PE}$ & Processing entity \\
\hline Representation & Describes the underlying geometric structure of data, combination of dimensions \\
\hline SMCL & Submodel core layer \\
\hline SMIL & Submodel interface layer \\
\hline Submodel & A tool or extension, which is plugged in via the MESSy interface \\
\hline SWMF & Space Weather Modeling Framework \\
\hline VISOP & VIsual Satellite OPerator submodel \\
\hline YAC & Yet Another Coupler \\
\hline
\end{tabular}




\section{The Supplement related to this article is available online at doi:10.5194/gmd-9-3639-2016-supplement.}

Acknowledgements. This work has been funded by the German Federal Ministry of Education and Research (BMBF) in the $\mathrm{HD}(\mathrm{CP})^{2}$ (High Definition Clouds and Precipitation for advancing Climate Prediction) initiative as Research for Sustainable Development (FONA, http://www.fona.de/) under grant no. 01LK1213C. Parts of this work have been supported by the European Union via funding of the project IS-ENES2 (grant agreement no. 312979).

We thank P. Adamidis, A. Dipankar, T. Jahns, M. Hanke, the staff at DKRZ, and the ICON developers for their support. We thank C. Meyer and L. Hoffmann for their support during the porting of our developments to JUQUEEN. We are grateful to L. Scheck for the provision of his code of VISOP and to J. Quaas for the provision of his jPDF code and the support during the development of GRAGG. We thank A. Pfeiffer for the fruitful discussion and the internal review, and A. Kerkweg for the comments on our manuscript.

The authors gratefully acknowledge the Deutsches Klimarechenzentrum (DKRZ) for providing computational resources on the HLRE-2 supercomputer, the HLRE-3 migration system, and the HLRE-3 supercomputer, as well as for technical support during the project.

The authors gratefully acknowledge the Gauss Centre for Supercomputing (GCS) for providing computing time on the GCS supercomputer SuperMUC at Leibniz Supercomputing Centre (LRZ, http://www.lrz.de) and through the John von Neumann Institute for Computing (NIC) on the GCS share of the supercomputer JUQUEEN at Jülich Supercomputing Centre (JSC). GCS is the alliance of the three national supercomputing centres HLRS (Universität Stuttgart), JSC (Forschungszentrum Jülich), and LRZ (Bayerische Akademie der Wissenschaften), funded by the German Federal Ministry of Education and Research (BMBF) and the German State Ministries for Research of Baden-Württemberg (MWK), Bavaria (StMWFK), and North Rhine-Westphalia (MIWF).

The authors wish to acknowledge use of the Ferret program for analysis and graphics in this paper. Ferret is a product of NOAA's Pacific Marine Environmental Laboratory, information is available at http://ferret.pmel.noaa.gov/Ferret/.

We thank Ilja J. Honkonen and one anonymous referee for their comments and valuable input to our manuscript.

The article processing charges for this open-access publication were covered by a Research

Centre of the Helmholtz Association.

Edited by: S. Easterbrook

Reviewed by: I. Honkonen and one anonymous referee

\section{References}

Ali, N., Carns, P., Iskra, K., Kimpe, D., Lang, S., Latham, R., Ross, R., Ward, L., and Sadayappan, P.: Scalable I/O forwarding framework for high-performance computing systems, in: 2009 IEEE International Conference on Cluster Computing and Workshops, 1-10, doi:10.1109/CLUSTR.2009.5289188, 2009.
Baker, A. H., Hammerling, D. M., Mickleson, S. A., Xu, H., Stolpe, M. B., Naveau, P., Sanderson, B., Ebert-Uphoff, I., Samarasinghe, S., De Simone, F., Carbone, F., Gencarelli, C. N., Dennis, J. M., Kay, J. E., and Lindstrom, P.: Evaluating Lossy Data Compression on Climate Simulation Data within a Large Ensemble, Geosci. Model Dev. Discuss., doi:10.5194/gmd-2016-146, in review, 2016.

Baumgaertner, A. J. G., Jöckel, P., Kerkweg, A., Sander, R., and Tost, H.: Implementation of the Community Earth System Model (CESM) version 1.2.1 as a new base model into version 2.50 of the MESSy framework, Geosci. Model Dev., 9, 125-135, doi:10.5194/gmd-9-125-2016, 2016.

Bodas-Salcedo, A., Webb, M. J., Brooks, M. E., Ringer, M. A., Williams, K. D., Milton, S. F., and Wilson, D. R.: Evaluating cloud systems in the Met Office global forecast model using simulated CloudSat radar reflectivities, J. Geophys. Res.-Atmos., 113, D00A13, doi:10.1029/2007JD009620, 2008.

Buehler, S. and Russchenberg, H. (Eds.): $\mathrm{HD}(\mathrm{CP})^{2}$ Observational Prototype Experiment, Atmos. Chem. Phys., http://www. atmos-chem-phys.net/special_issue366.html, 2014.

Collins, N., Theurich, G., DeLuca, C., Suarez, M., Trayanov, A., Balaji, V., Li, P., Yang, W., Hill, C., and da Silva, A.: Design and Implementation of Components in the Earth System Modeling Framework, Int. J. High Perform. C., 19, 341-350, doi:10.1177/1094342005056120, 2005.

Collins, W. D., Bitz, C. M., Blackmon, M. L., Bonan, G. B., Bretherton, C. S., Carton, J. A., Chang, P., Doney, S. C., Hack, J. J., Henderson, T. B., Kiehl, J. T., Large, W. G., McKenna, D. S., Santer, B. D., and Smith, R. D.: The Community Climate System Model Version 3 (CCSM3), J. Climate, 19, 2122-2143, doi:10.1175/JCLI3761.1, 2006.

Craig, A. P., Jacob, R., Kauffman, B., Bettge, T., Larson, J., Ong, E., Ding, C., and He, Y.: CPL6: The New Extensible, High Performance Parallel Coupler for the Community Climate System Model, Int. J. High Perform. C., 19, 309-327, doi:10.1177/1094342005056117, 2005.

Dipankar, A., Stevens, B., Heinze, R., Moseley, C., Zängl, G., Giorgetta, M., and Brdar, S.: Large eddy simulation using the general circulation model ICON, J. Adv. Model. Earth Syst., 7, 963-986, doi:10.1002/2015MS000431, 2015.

Gent, P. R., Danabasoglu, G., Donner, L. J., Holland, M. M., Hunke, E. C., Jayne, S. R., Lawrence, D. M., Neale, R. B., Rasch, P. J., Vertenstein, M., Worley, P. H., Yang, Z.-L., and Zhang, M.: The Community Climate System Model Version 4, J. Climate, 24, 4973-4991, doi:10.1175/2011JCLI4083.1, 2011.

Hanke, M., Redler, R., Holfeld, T., and Yastremsky, M.: YAC 1.2.0: new aspects for coupling software in Earth system modelling, Geosci. Model Dev., 9, 2755-2769, doi:10.5194/gmd-9-27552016, 2016.

Hoppe, C. M., Hoffmann, L., Konopka, P., Grooß, J.-U., Ploeger, F., Günther, G., Jöckel, P., and Müller, R.: The implementation of the CLaMS Lagrangian transport core into the chemistry climate model EMAC 2.40.1: application on age of air and transport of long-lived trace species, Geosci. Model Dev., 7, 2639-2651, doi:10.5194/gmd-7-2639-2014, 2014.

Jöckel, P.: Earth System Modeling, in: Atmospheric Physics, edited by: Schumann, U., chap. 35, 577-590, Springer, Berlin Heidelberg, Germany, doi:10.1007/978-3-642-30183-4_35, 2012. 
Jöckel, P., Sander, R., Kerkweg, A., Tost, H., and Lelieveld, J.: Technical Note: The Modular Earth Submodel System (MESSy) - a new approach towards Earth System Modeling, Atmos. Chem. Phys., 5, 433-444, doi:10.5194/acp-5-433-2005, 2005.

Jöckel, P., Tost, H., Pozzer, A., Brühl, C., Buchholz, J., Ganzeveld, L., Hoor, P., Kerkweg, A., Lawrence, M. G., Sander, R., Steil, B., Stiller, G., Tanarhte, M., Taraborrelli, D., van Aardenne, J., and Lelieveld, J.: The atmospheric chemistry general circulation model ECHAM5/MESSy1: consistent simulation of ozone from the surface to the mesosphere, Atmos. Chem. Phys., 6, 50675104, doi:10.5194/acp-6-5067-2006, 2006.

Jöckel, P., Kerkweg, A., Pozzer, A., Sander, R., Tost, H., Riede, H., Baumgaertner, A., Gromov, S., and Kern, B.: Development cycle 2 of the Modular Earth Submodel System (MESSy2), Geosci. Model Dev., 3, 717-752, doi:10.5194/gmd-3-717-2010, 2010.

Kerkweg, A. and Jöckel, P.: The 1-way on-line coupled atmospheric chemistry model system $\operatorname{MECO}(\mathrm{n})$ - Part 1: Description of the limited-area atmospheric chemistry model COSMO/MESSy, Geosci. Model Dev., 5, 87-110, doi:10.5194/gmd-5-87-2012, 2012a.

Kerkweg, A. and Jöckel, P.: The 1-way on-line coupled atmospheric chemistry model system MECO(n) - Part 2: On-line coupling with the Multi-Model-Driver (MMD), Geosci. Model Dev., 5, 111-128, doi:10.5194/gmd-5-111-2012, 2012 b.

Kerkweg, A. and Jöckel, P.: The infrastructure MESSy submodels GRID (v1.0) and IMPORT (v1.0), Geosci. Model Dev. Discuss., 8, 8607-8633, doi:10.5194/gmdd-8-8607-2015, 2015.

Kuhn, M., Kunkel, J., and Ludwig, T.: Data Compression for Climate Data, Supercomputing Frontiers and Innovations, 3, 75-94, doi:10.14529/jsfi160105, 2016.

Kunkel, J., Kuhn, M., and Ludwig, T.: Exascale Storage Systems An Analytical Study of Expenses, Supercomputing Frontiers and Innovations, 1, 116-134, doi:10.14529/jsfi140106, 2014.

Pozzer, A., Jöckel, P., Kern, B., and Haak, H.: The AtmosphereOcean General Circulation Model EMAC-MPIOM, Geosci. Model Dev., 4, 771-784, doi:10.5194/gmd-4-771-2011, 2011.
Redler, R., Valcke, S., and Ritzdorf, H.: OASIS4 - a coupling software for next generation earth system modelling, Geosci. Model Dev., 3, 87-104, doi:10.5194/gmd-3-87-2010, 2010.

Roeckner, E., Brokopf, R., Esch, M., Giorgetta, M., Hagemann, S., Kornblueh, L., Manzini, E., Schlese, U., and Schulzweida, U.: Sensitivity of Simulated Climate to Horizontal and Vertical Resolution in the ECHAM5 Atmosphere Model, J. Climate, 19, 3771-3791, doi:10.1175/JCLI3824.1, 2006.

Scheck, L., Frèrebeau, P., Buras-Schnell, R., and Mayer, B.: A fast radiative transfer method for the simulation of visible satellite imagery, J. Quant. Spectrosc. Ra., 175, 54-67, doi:10.1016/j.jqsrt.2016.02.008, 2016.

Tóth, G., Sokolov, I. V., Gombosi, T. I., Chesney, D. R., Clauer, C. R., De Zeeuw, D. L., Hansen, K. C., Kane, K. J., Manchester, W. B., Oehmke, R. C., Powell, K. G., Ridley, A. J., Roussev, I. I., Stout, Q. F., Volberg, O., Wolf, R. A., Sazykin, S., Chan, A., Yu, B., and Kóta, J.: Space Weather Modeling Framework: A new tool for the space science community, J. Geophys. Res.-Space, 110, A12226, doi:10.1029/2005JA011126, 2005.

Valcke, S.: The OASIS3 coupler: a European climate modelling community software, Geosci. Model Dev., 6, 373-388, doi:10.5194/gmd-6-373-2013, 2013.

Valcke, S., Guilyardi, E., and Larsson, C.: PRISM and ENES: a European approach to Earth system modelling, Concurr. Comp.Pract. E., 18, 247-262, doi:10.1002/cpe.915, 2006.

Valcke, S., Balaji, V., Craig, A., DeLuca, C., Dunlap, R., Ford, R. W., Jacob, R., Larson, J., O'Kuinghttons, R., Riley, G. D., and Vertenstein, M.: Coupling technologies for Earth System Modelling, Geosci. Model Dev., 5, 1589-1596, doi:10.5194/gmd-51589-2012, 2012.

Zängl, G., Reinert, D., Rípodas, P., and Baldauf, M.: The ICON (ICOsahedral Non-hydrostatic) modelling framework of DWD and MPI-M: Description of the non-hydrostatic dynamical core, Q. J. Roy. Meteor. Soc., 141, 563-579, doi:10.1002/qj.2378, 2015. 